\title{
Anabases
}

ANABASES Traditions et réceptions de l'Antiquité

$16 \mid 2012$

Varia

\section{Jugurtha héros national : jalons sur un itinéraire}

\section{Jacques Alexandropoulos}

\section{OpenEdition}

\section{Journals}

Édition électronique

URL : http://journals.openedition.org/anabases/3872

DOI : 10.4000/anabases.3872

ISSN : 2256-9421

\section{Éditeur}

E.R.A.S.M.E.

\section{Édition imprimée}

Date de publication : 1 octobre 2012

Pagination : 11-29

ISSN : 1774-4296

\section{Référence électronique}

Jacques Alexandropoulos, « Jugurtha héros national : jalons sur un itinéraire », Anabases [En ligne],

16 | 2012, mis en ligne le 01 octobre 2015, consulté le 10 décembre 2020. URL : http://

journals.openedition.org/anabases/3872 ; DOI : https://doi.org/10.4000/anabases.3872 
Anabases 16 (2012), p. 11-29.

\section{Jugurtha héros national : jalons sur un itinéraire}

JacQues Alexandropoulos

D ANS UN LONG ARTICle intitulé "De l'éternel Méditerranéen à l'éternel Jugurtha ", paru en $1982^{1}$, Jean Déjeux revient sur le rôle et l'affrontement des différents " mythes et contre-mythes » créés par les colonisateurs et les colonisés autour de la question algérienne. Au thème de l'éternel Méditerranéen latin d'un Louis Bertrand vient s'opposer celui de l'éternel Jugurtha berbère de Jean Amrouche. J. Déjeux expose ainsi, en spécialiste des littératures maghrébines contemporaines, la dialectique de cet affrontement. La richesse de ce travail suggère aussitôt à l'antiquisant de prolonger à sa façon la réflexion en reliant le mythe contemporain à ses sources antiques par un retour sur l'itinéraire de Jugurtha héros national, depuis le texte fondateur de Salluste jusqu'aux réélaborations contemporaines les plus achevées, celle de Jean Amrouche en 1943 et de Mohammed Chérif Sahli en 1946. S'il ne peut s'agir, compte tenu de l'extrême richesse du thème, que de quelques sondages dans le vaste champ d'études de la récupération coloniale et postcoloniale de l'Antiquité, au moins cela servira-t-il à mettre en évidence un cas de particulière efficience de l'idéologie coloniale appuyée sur l'autorité des Anciens ainsi que de la réaction suscitée en retour.

Les origines de la guerre de Jugurtha remontent à la querelle de succession ouverte par la mort du roi numide Micipsa en 118 av. J.-C. Trois princes briguent le trône, les deux fils du roi, Adherbal et Hiempsal, et un neveu de naissance illégitime mais beaucoup plus talentueux, Jugurtha, qui élimine les deux autres par la ruse et la violence. Corrompue par Jugurtha, l'aristocratie romaine se cantonne dans une "neutralité " favorable à ce dernier, jusqu'au moment où le prince commet l'erreur de massacrer des commerçants italiens. La plèbe de Rome pousse alors à la guerre, une longue et dure

1 Dans les Studi maghrebini, volume XIV, 1982, p. 67-162. 
guerre de six ans au bout de laquelle Jugurtha est capturé par traîtrise et livré à Marius par le roi de Maurétanie, Bocchus. C'est un peu plus d'une soixantaine d'années après la fin de la guerre que Salluste rédige l'ouvrage qui constitue quasiment notre seule source sur le prince numide ${ }^{2}$. En ce sens il est bien le créateur absolu de Jugurtha; c'est lui qui en construit le personnage sans que nous ayons la possibilité, sauf découverte archéologique révolutionnaire, d'en dresser un contre-portrait à partir de sources internes. Nous en sommes réduits à suivre le regard de Salluste avec comme seules défenses celles de la critique des textes. On remarquera que Jugurtha est, de ce point de vue et malgré les apparences, le plus mal loti des grands souverains berbères. En effet, s'il est le seul à bénéficier d'une quasi-biographie quasi-contemporaine, il n'a en revanche laissé, contrairement aux autres, aucun discours propre. On entend par discours propre, sinon des écrits qui nous seraient miraculeusement parvenus, du moins des inscriptions ou des émissions monétaires qui, correctement interprétées, pourraient nous permettre de reconstituer un tant soit peu son message politique. Or, curieusement, Jugurtha, quoi qu'on ait pu en dire, ne nous a laissé aucune émission monétaire comme nous le verrons plus loin. Salluste constitue donc bien l'alpha et l'oméga de nos sources.

Or le Bellum iugurthinum présente des singularités souvent relevées, qui compliquent particulièrement le travail d'approche de Jugurtha par l'historien et facilitent d'autant la construction du mythe. Ainsi, la chronologie de la guerre reste très obscure dans le détail. Certes, nous connaissons les commandants en chef successifs du " corps expéditionnaire » romain, en pouvant reconstituer, mais non pas grâce à Salluste, les dates approximatives de leur entrée en fonctions. Mais, au-delà de ces cadres très généraux, la chronologie des épisodes n'est pas indiquée, et la seule date précise que Salluste nous donne est erronée ${ }^{3}$. C'est donc d'un flou chronologique particulier qu'émergent d'autant plus vigoureusement les épisodes de combat et l'image des personnages-clefs : Jugurtha lui-même, Métellus, Marius et les membres éminents de l'aristocratie romaine.

Si le temps reste approximatif dans l'œuvre de Salluste, il en va de même de l'espace. Où s'est déroulée la guerre de Jugurtha ? R. Syme rappelle que l'on ne peut relever dans le récit, pour six années de campagne, que neuf indications de villes et trois de fleu-

2 Pour le texte : Salluste, Catilina, Jugurtha, Fragments des Histoires, éd. A. ERNout, Paris, Les Belles Lettres, 1941 ; sur les autres sources, très secondaires, et les conditions de rédaction du texte : S. Gsell, Histoire ancienne de l'Afrique du Nord, Paris, Hachette, 1913-1928, VII, p. 123-134.

3 Voir déjà Th. Mommsen, Histoire romaine, trad. C. A. AleXAndre, éd. C. Nicolet, Paris, Laffont, 1985 , p. 814, la longue note 1 sur les incertitudes chronologiques dans le B.J., "La chronologie a été négligée plus que de raison ". Même remarque chez S. GseLL, Histoire ancienne de l'Afrique du Nord, VII, p. 130 : "La chronologie étant sacrifiée ou devant se soumettre aux convenances de la composition "; R. SYME, Sallust, Berkeley, 1964, traduction P. RoBin, Paris, Les Belles Lettres, 1983, p. 123 sqq. sur la chronologie en général et les problèmes liés à la mention de la date de 109 pour l'attaque de Spurius Albinus. 
ves, et encore certaines d'entre elles sont-elles pour nous impossibles à localiser ou très discutées ${ }^{4}$. Reste-t-il au moins un cadre général ? Ce dernier même demeure imprécis au point que le Bellum iugurthinum a pu donner lieu à un essai déconcertant de recomposition drastique de nos conceptions géographiques de l'Afrique antique. L'archiviste A. Berthier, établi à Constantine et de ce fait sensible aux réalités géographiques locales, s'était attaché à reprendre les difficultés posées par la géographie de Salluste. Ainsi, la ville de Cirta (Constantine), ce nid d'aigle qui domine les légendaires gorges du Rhummel, semble présentée comme une ville de plaine ${ }^{5}$. Il en va de même de Zama, récemment identifiée à l'actuelle Jama ${ }^{6}$. Par ailleurs, certains déplacements de troupes romaines, tels qu'ils sont décrits par Salluste, déroutent le lecteur : ainsi lorsque l'auteur juxtapose quasiment la prise d'une ville du sud tunisien et l'attaque d'un fortin que l'on devrait situer aux actuels confins algéro-marocains. S. Gsell en avait été réduit à supposer que Salluste avait négligé d'indiquer qu'un hiver s'est écoulé entre les deux faits ${ }^{7}$ ! On sait que tout cela a amené $\mathrm{A}$. Berthier à proposer une redéfinition drastique de termes aussi généraux que ceux de "Numidie " ou de "Maurétanie ${ }^{8}$ ». La Maurétanie se trouve ramenée du Maroc actuel à l'Aurès, et le terme "Numidie », au lieu de recouvrir l'essentiel de l'Algérie contemporaine, ne désignerait plus que le nord-ouest tunisien. La guerre de Jugurtha ne se serait plus déployée sur la majeure partie du Maghreb, mais uniquement dans une zone territoriale comprise entre Constantine et l'Aurès en Algérie, Le Kef et Gafsa en Tunisie. Ces théories ont été plus que fraîchement accueillies, donnant lieu à de sévères mises au point. Ce que l'on retiendra de la querelle, c'est le flou exemplaire de la géographie de Salluste, aussi remarquable que son imprécision chronologique. Et cela quoi qu'en ait pensé notre historien, qui jugeait en avoir assez dit sur ce point pour l'édification de son

4 Les neuf villes en question s'entendent en dehors de celles (6) qui apparaissent comme de grands repères connus de tous : Cirta, Hippone, Carthage, Utique, Hadrumète et Leptis. Sur l'imprécision géographique de Salluste dans le B.J. : S. GSELL, Histoire ancienne de l'Afrique du Nord, p. 124-125 ; R. Syme, Sallust, p. 126 sqq. ; E. Tiffou, "Salluste et la géographie ", Mélanges R. Dion, Caesarodunum, IX bis (1974), p. 151-160 ; et surtout la querelle déclenchée par A. BERTHIER, voir infra.

$5 B$. J., XXIV, où Salluste indique que Jugurtha entoure la ville d'un fossé et de divers moyens de siège.

6 B.J., LVII, 1. Sur l'identification : A. Ferjaour, "Localisation de Zama regia à Jama ", Comptes rendus des séances de l'AIBL (2002), vol. 146, n 3, p. 1003-1017.

$7 \quad$ B.J., XCII ; S. Gsell, Histoire ancienne de l'Afrique du Nord, VII, p. 235.

8 A. Berthier, R. Charlier, J. Juillet, « Le Bellum iugurthinum de Salluste et le problème de Cirta ", RSAC 67 (1950-51), p. 1-150 ; repris dans A. BERTHIER, " Note sur l'épigraphie du Kef », RSAC 68 (1953), p. 175-198 ; "Colonia Cirta Sittianorum », RSAC 70 (195758-59), p. 91-118 et La Numidie, Paris, Picard, 1981, en particulier p. 71-79. Parmi les réactions résolument défavorables, "Compte rendu de l'ouvrage de MM. Berthier, Juillet et Charlier ", RA (1951), XXXVII, p. 248-250 et J. DesangeS, "Compte rendu de l'ouvrage de M. Berthier", REL (1982), p. 570-572. 
lecteur ${ }^{9}$. Jugurtha est donc capable de se mouvoir hors de certaines contraintes de l'espace et du temps. Seuls comptent son caractère et son action.

Toutes ces imprécisions font ressortir par contrecoup la vigueur des portraits sallustéens, et Jugurtha est dépeint avec une puissance souvent notée ${ }^{10}$. Cela étant, de quelle manière la construction du personnage par Salluste prépare-t-elle l'avènement futur du héros national ?

Jugurtha relève, comme son entourage et sa région d'origine, d'un ensemble de stéréotypes liés aux barbares africains, habituels dans l'historiographie romaine et largement connus. Chez Salluste, bien des traits du caractère africain peuvent se résumer dans le terme latin de mobilitas ${ }^{11}$. Ce mot revient souvent dans le texte, avec ses synonymes et dérivés pour désigner aussi bien certains aspects du caractère de Jugurtha que des Numides en général. Cette "mobilité " qui apparaît comme la caractéristique essentielle du Numide dont Jugurtha devient l'incarnation, peut signifier agilité d'esprit, adaptation, souplesse, mais aussi désorganisation, versatilité, instabilité, ruse et déloyauté. Sur le plan de l'action, elle explique une rapidité exceptionnelle du roi et des Numides, leur capacité à se jouer de l'armée romaine, à apparaître et à disparaître dans un milieu naturel et humain complice. Au plan de la civilisation, elle rejoint l'idée de nomadisme par opposition au système de la cité où peut s'épanouir la constantia romaine. Elle participe ainsi de l'essence même de l'Africain dont elle fait pour le conquérant un être insaisissable dans son mode de fonctionnement et comme objet de conquête. Et de fait, c'est par la versatilité et la ruse d'un autre Africain, le roi Bocchus de Maurétanie, que Rome aura raison de Jugurtha. L'Afrique, au bout du compte, s'est prise au piège de sa propre mobilitas! Cette perspective essentialiste est la contribution de Salluste à la fondation de l'imaginaire collectif de la conquête romaine, qui annonce l'établissement du nouvel ordre impérial augustéen ${ }^{12}$. Sa contribution aussi et du

9 On voit revenir des notations vagues, comme " (Metellus) brûle nombre de forteresses " $(\mathrm{LIV}, 6)$; "Le consul marche sur les places et les châteaux forts " (LXXXIX, 1) ; "Le consul marche sur d'autres places» (XCII, 3). Et par ailleurs, il conclut un exposé sur la géographie générale de l'Afrique par : "Ces quelques notes sur l'Afrique et ses habitants suffisent aux exigences de mon sujet " (XIX, 8).

10 Voir entre autres les remarques de S. Gsell, Histoire ancienne de l'Afrique du Nord, VII, p. 130 : "Le Jugurtha est un véritable drame où quelques scènes choisies se détachent en haut-relief, les épisodes intermédiaires étant négligés ou même complètement omis, la chronologie étant sacrifiée ou devant se soumettre aux convenances de la composition, la géographie étant réduite au strict nécessaire pour situer ces scènes dans l'espace. "

11 "Genus Numidarum infidum, ingenio mobili... ", (XLVI) ; " ... tanta mobilitate sese Numidae gerunt...", (LVI) ; Bocchus : "id simulaveritne... an mobilitate ingeni..." ", (LXXXIX) ; " ... regiae voluntates ut vehementes sic mobiles... ", (CXIII).

12 Parmi une très abondante bibliographie, A. Momigliano, Sagesses barbares. Les limites de l'hellénisation, Paris, Maspero, 1979 ; J.P.V.D. BALSDON, Romans and Aliens, Londres, Duckworth, 1979 ; J.-A. DAUge, Le Barbare. Recherches sur la conception romaine de la barbarie et de la civilisation, coll. "Latomus ", vol. 176, Bruxelles, 1981 ; Michel 
même coup, à plus long terme et par analogie, à la formation de l'imaginaire impérial et colonial français contemporain.

Mais à plus longue échéance encore, et selon d'autres modalités que nous verrons, Salluste a préparé aussi le Jugurtha du nationalisme algérien. Et sur ce point, on fait évidemment le rapprochement entre ce héros sur-caractérisé et un Vercingétorix créé dans des conditions assez similaires et littérairement quasi contemporaines, par un conquérant dont le récit est guidé par des considérations de politique purement romaine ${ }^{13}$. Comme Vercingétorix, Jugurtha a connu le succès, puis, tout aussi importante, la défaite. On sait que les héros nationaux sont souvent des vaincus dans la mesure où la défaite même joue un rôle fondateur dans la construction des identités nationales. S’y référer permet de prouver l'existence de la communauté nationale avant même sa reconnaissance officielle, tout en expliquant par la lutte de libération nationale le long temps qui peut s'écouler jusqu'à l'avènement de l'État-nation correspondant ${ }^{14}$.

Après avoir souligné en quoi le récit de Salluste offrait une matière de choix pour une récupération mythique contemporaine, on peut rappeler certains des écarts entre ces mythes aboutis et la source romaine. Et d'abord, l'Afrique n'est pas la préoccupation première de Salluste.

L'historien l'indique nettement au début de son ouvrage. S'il raconte cette guerre, c'est « d'abord qu' elle fut rude et acharnée, mêlée de succès et de revers, ensuite parce que c'est alors pour la première fois qu'on osa marcher contre l'insolence de la noblesse $^{15}$ ». La hiérarchisation des deux points d'intérêt est claire. Il y a, certes, les péripéties de l'événementiel, et c'est là la part proprement africaine de l'ouvrage. Quelle qu'en soit l'importance pour la conscience impériale romaine, l'essentiel reste, pour le césarien jadis engagé dans la politique qu'était Salluste, le rôle de ce conflit dans l'engrenage qui a mené au cataclysme des guerres civiles. Elles sont loin d'être terminées lorsque Salluste écrit, et c'est là une préoccupation essentielle, qui nourrit sa réflexion d'historien et son légendaire " pessimisme ". L'Afrique n'est donc pas sa préoccupation première, et cela nous invite à relativiser d'autant plus certains aspects de son Jugurtha. Celui-ci n'est pas qu'un prince numide, mais aussi une sorte de double de Catilina. Il est, comme lui, à la fois proche et loin du pouvoir, doté de qualités exceptionnelles

Dubuisson, "La vision romaine de l'étranger - Stéréotypes, idéologie et mentalités ", www.class.ulg.ac.be/ressources/vision.

13 Ch. Amalvi, De l'art et la manière d'accommoder les héros de l'histoire de France, Paris, Albin Michel, 1988, p. 53-87 ; Ch. GoudinEau, Le dossier Vercingétorix, Arles, Actes Sud/ Errance, 2001, première partie : "Naissance, vie et crépuscule d'un mythe "; M. REDDÉ, "Alésia et la mémoire nationale française ", Anabases 9 (2009), p. 13-24.

14 "Une défaite illustre... l'hypothèse de base des nationalismes modernes : l'antériorité logique et chronologique de la nation par rapport à l'État»: J.-P. AlberT, « Pourquoi les héros nationaux sont-ils souvent des vaincus ? ", in P. Cabanel et P. Laborie éd., Penser la défaite, Toulouse, Privat, 2002, p. 25.

15 B.J., V, 1. 
qu'il dévoie en prenant des chemins de traverse. Les deux personnages rappellent tous ceux qui, par leur course au pouvoir, mènent leur communauté à une lutte fratricide. Cette perspective proprement romaine est évidement sans intérêt pour la construction des mythes contemporains, mais elle pointe l'écart entre le mythe et sa source antique, et met ainsi d'autant mieux en valeur l'accentuation qu'il effectue sur un autre point, celui de la critique de l'impérialisme romain.

On voit en effet, chez Salluste, Jugurtha lancer des invectives contre l'expansion militaire de Rome ${ }^{16}$. Ce passage fait écho à un autre texte sallustéen plus développé et célèbre : la lettre de Mithridate au roi des Parthes, qui se présente comme un réquisitoire en règle contre l'impérialisme romain. Nul doute que cette page du Bellum iugurthinum ait contribué à donner à Jugurtha sa stature de héros national, mais le lien entre les deux passages montre, comme on le sait, qu'il faut plus y voir un " topos " et une attaque répétée de Salluste contre la gestion de la conquête par l'oligarchie sénatoriale qu'une véritable critique de l'expansionnisme romain reproduisant un authentique discours de Jugurtha. Le roi ne fait ici que prêter sa voix aux critiques de l'historien romain ${ }^{17}$. Il n'en reste pas moins que Salluste en fait dire assez à son Jugurtha pour le promettre à un bel avenir de champion de la liberté numide.

La fécondité du personnage sallustéen dans la perspective d'une construction mythique et d'une récupération analogique se trouve donc à la confluence de plusieurs éléments. Donné par Salluste comme l'incarnation du caractère numide, il constitue d'abord un archétype qui servira plus tard d'assise au stéréotype colonial du Berbère. Il est d'autre part vaincu après une guerre acharnée contre les futurs maîtres de l'Afrique du Nord, les Romains, dont se réclameront ensuite les conquérants français du Maghreb. Par ailleurs, son épopée, du fait de Salluste, jaillit comme une brusque et violente lumière, d'un contexte historique africain qui reste très obscur. Installé enfin par un conteur exceptionnel en plein centre d'un récit à la fois imprécis et haut en couleur, il reçoit du génie littéraire de Salluste un surcroît de force. Car on a maintes fois souligné, chez notre historien, une puissance de plume, des capacités de mise en scène des personnages, de suggestion des situations et des paysages qui relèvent d'un

16 B.J., XLIX : « Il (Jugurtha) les (les Numides) encourage, et les conjure de se rappeler leur antique valeur, leur victoire passée, et de défendre leur royaume et leur roi contre l'avarice des Romains. » Voir surtout le discours de Jugurtha au roi Bocchus (LXXXI) : «Les Romains... sont un peuple injuste, d'une cupidité sans bornes, ennemi de tout le genre humain ; ils ont pour lui faire la guerre le même motif qui les a armés contre lui-même et tant d'autres nations, leur besoin de dominer, qui en fait les adversaires de tous les empires. Aujourd'hui c'est lui-même, hier c'étaient les Carthaginois, et le roi Persée, demain ce sera quiconque leur paraîtra le plus riche qui sera leur ennemi "; à rapprocher de la lettre VI des Epistulae et orationes.

17 Sur ces points, R. Syme, Sallust, p. 204 sqq. et dernièrement E. Adler, Valorizing the Barbarians : Enemy Speeches in Roman Historiography. Ashley and Peter Larkin series in Greek and Roman culture, Austin, University of Texas Press, 2011. 
art consommé du récit ${ }^{18}$. Une narration qui reste par ailleurs, et cela lui donne encore plus de force, notre seule source sur le sujet ${ }^{19}$.

Mais quand et comment donc Jugurtha devient-il véritablement un héros national ? Sa " consécration » en ce sens n'attend pas en fait la lutte des partis nationalistes maghrébins pour l'indépendance et ne vient pas des milieux colonisés. On peut en trouver une première étape l'année même de la conquête de l'Algérie, dans un cours prononcé par Michelet à l'École Normale Supérieure en 1829-1830 et qui deviendra l'Histoire romaine, publiée en 1831.

Michelet introduit, sans surprise, la lecture révolutionnaire et nationale du héros. Il ne consacre que quelques pages à Jugurtha, dans lesquelles la part belle est faite, comme chez Salluste, aux aspects proprement romains de l'épisode. Néanmoins, il en dit bien assez sur Jugurtha pour que nous puissions aussitôt saisir la distorsion qu'il fait subir à sa source latine en inversant la hiérarchie des buts de guerre du roi. Chez Salluste, le prince écarté a priori de la succession par sa naissance illégitime cherche avant tout le pouvoir suprême en Numidie ; c'est en second lieu seulement, comme argument mobilisateur, qu'il utilise dans un discours le thème de la défense de la liberté contre Rome qui permet surtout à Salluste de charger l'aristocratie romaine. Michelet inverse l'ordre des priorités et l'annonce d'ailleurs d'emblée : «On a regardé Jugurtha comme un usurpateur, il aurait fallu s'informer d'abord s'il existait une loi d'hérédité dans les déserts de Numidie... Les Numides pensèrent que la volonté d'un mort ne pouvait prévaloir sur le droit de la nation ${ }^{20}$. " La lutte prend alors une tournure essentiellement nationale. Lorsque Jugurtha élimine son frère Adherbal, il supprime le « candidat antinational » qui constitue « le dernier obstacle à l'unité de la Numidie ». Il est le "vrai Numide désigné au trône par la voix des Numides ", et donc titulaire d'une double légitimité, celle d'incarnation de son peuple et de candidat désigné en quelque sorte par le suffrage universel. Rien d'étonnant donc à ce que, semblables aux Français de l'An II, les Numides face à un ennemi pratiquant " une guerre d'extermination " et venant " égorger dans toutes les villes tous les mâles en âge de puberté », se dressent pour "soutenir avec une héroïque obstination le chef qu'ils s'étaient donné ». Les

18 R. Syme, Sallust, p. 127, relève la valeur pittoresque de l'Afrique chez Salluste en s'inquiétant de sa valeur historique : "Salluste présente une peinture de la guerre d'Afrique valable à toute époque. Des formules vigoureuses évoquent le désert, le maquis, le terrain inégal, l'ennemi qui se dérobe, la soif et la fatigue, la trahison et le meurtre. En ce domaine les mérites de Salluste sont manifestes et reconnus. Il reste à se demander s'il a mis convenablement en lumière le caractère spécifique de cette guerre : causes, durée et conclusion. "

19 On peut citer ici S. Gsell, Histoire ancienne de l'Afrique du Nord, VII, p. 122 : "Dans l'obscurité qui enveloppe l'Afrique du Nord entre la destruction de Carthage et la campagne de Jules César, la figure de Jugurtha apparaît, entourée de l'éclat que lui donne l'œuvre célèbre de Salluste."

20 J. Michelet, Histoire romaine, éd. P. Petitier, Paris, Les Belles Lettres, 2003, p. 389-394; p. 390 pour la citation. 
références nationales et révolutionnaires orientent ici la relecture du texte de Salluste, modifiant le sens de la lutte de Jugurtha, et par là-même la stature du personnage dont le combat personnel pour le trône de Numidie devient lutte nationale contre l'envahisseur romain à l'image de ce qui s'écrivait alors pour la Gaule ${ }^{21}$.

Il reste, par les vertus de l'analogie, à revivifier, réactualiser ce héros, à achever de le "nationaliser" en lui trouvant un double contemporain ${ }^{22}$. Il faut pour cela une méthode et le double en question. En ce qui concerne la méthode, l'historiographie coloniale à ses débuts approche le personnage de Jugurtha dans la tradition bien connue de fidélité absolue à la lettre des textes anciens, illustrée entre autres par le mot de Fustel de Coulanges : «Le meilleur des historiens est celui qui se tient le plus près des textes, qui n'écrit et même ne pense que d'après eux ${ }^{23}$. "Cette attitude, qui recoupe le souci du Ministère de la Guerre de s'appuyer sur l'expérience des Anciens pour conquérir et coloniser l'Algérie, amène à déchiffrer les réalités contemporaines à travers le regard de ces autorités, jusqu'à fusionner dans l'écriture les mots de jadis et ceux du moment, dans une clarification et légitimation réciproques du passé et du présent. Il s'agit dès lors et par ce biais, pour toute une lignée d'historiens comme S. Gsell, de prouver, outre la validité de leur discours scientifique, leur fidélité à la culture classique dont ils sont les héritiers, les représentants et les maîtres d'application dans l'œuvre en cours en terre coloniale. Il en sortira une vision de Jugurtha et des Africains, antiques comme contemporains, très proche du stéréotype du Numide tel qu'il apparaît chez Salluste : mobile, versatile, rusé, déloyal, désorganisé et cruel $^{24}$. Tite-Live y rajoutait une sensualité immodérée ${ }^{25}$. On retrouve alors le Maghrébin intemporel dans le rôle de l'individu éternellement à civiliser par le non moins éternel civilisateur latin. Tout cela avec le risque évident, si l'on réunit cette approche avec celle de Michelet, d'offrir une construction idéologique parfaite aux mouvements nationalistes ${ }^{26}$.

21 On sait que Michelet s'était beaucoup inspiré d'Amédée Thierry (1797-1873) qui, dans son Histoire des Gaulois (1828), insistait à propos de la guerre contre César, sur l'opposition parmi les Gaulois entre un "parti romain » et un " parti national »; cf. Christian Goudineau, Le dossier Vercingétorix, p. 30 sqq.

22 Voir P. Centlivres, D. Fabre, F. Zonabend, La fabrique des héros, Collection Ethnologie de la France, cahier 12, Paris, MSH, 1998, et notamment la contribution de D. FABRE, "L'atelier des héros ", p. 233-309.

23 Histoire des institutions politiques de la France. La monarchie franque, Paris, Hachette, 1888 , p. 33.

24 S. Gsell, Histoire ancienne de l'Afrique du Nord, VI, p. 274 sqq.

25 Ainsi lorsque Massinissa succombe au charme de Sophonisbe « ut est genus Numidarum in Venerem praeceps ": Histoire romaine, XXX, 12, 18.

26 Jugurtha est entériné comme héros national berbère par l'historiographie coloniale, par des historiens aussi différents que S. GsELL («Salluste avait raconté la dernière grande lutte des Africains pour l'indépendance nationale »), Histoire ancienne de l'Afrique du Nord, p. 89, et Ch. A. Julien (« Mais les Numides ne perdaient pas le souvenir de l'aguellid qui 
Le double contemporain du roi numide s'impose assez rapidement, et la version en partie commune aux coloniaux et aux nationalistes, du Jugurtha " héros national " de Michelet et archétype colonial du Berbère intemporel, s'achève par sa superposition avec Abdelkader. Lorsque Michelet décrit Jugurtha, il le dépeint comme " ardent et intrépide " et surtout comme " le meilleur cavalier d'Afrique, le plus ardent chasseur, toujours le premier à frapper le lion ${ }^{27}$ ". Cette imagerie qui reprend celle de Salluste ${ }^{28}$, et liée par ailleurs à celle du souverain hellénistique et d'Alexandre le Grand, en annonce de nouvelles : celles du « cavalier d'Afrique », du « chasseur d'Afrique » et du chasseur de lion en Afrique, intimement liées à la conquête de l'Algérie dans la vision romantique qu'en avaient certains cercles d'officiers français, ce que Jacques Berque appelait « le romantisme des bureaux arabes ${ }^{29}$ ". C'est dans ces cercles que naît l'image d'un affrontement chevaleresque entre Abdelkader et les généraux français, cercles cultivés qui ont tôt fait de conférer à cet affrontement ses lettres de noblesse en effectuant le parallèle avec la guerre de Jugurtha. Dès 1843 le maréchal Bugeaud lui-même parlait du chef algérien comme "d'un homme de génie que l'histoire doit placer à côté de Jugurtha ${ }^{30}$ ". On pourrait citer de nombreux autres exemples ${ }^{31}$. Jugurtha a désormais repris vie, s'est réincarné, effectuant une médiation entre les époques, au moins du côté du colonisateur pour l'instant.

Le Jugurtha ainsi recréé aux débuts de la colonisation ne peut pas être exempt des ambiguïtés liées à sa constitution. Ses aspects nationaux et emblématiques peuvent bien servir le colonisateur si l'on considère qu'en se soumettant, son successeur Abdelkader

les avait conduits dans la lutte contre l'impérialisme romain "), Histoire de l'Afrique $d u$ Nord, Paris, Payot, 1930, p. 129-130.

27 Histoire romaine, p. 389-390.

28 B.J., VI : « ... equitare, jaculari... pleraque tempora in venando agere, leonem atque alias feras primus aut in primis ferire...".

29 "Cent-vingt-cinq ans de sociologie maghrébine ", Annales ESC, $11^{\mathrm{e}}$ année (juillet-septembre 1956), p. 299-301. L. LACROIX, Numidie et Maurétanie, in L'univers pittoresque, Afrique Ancienne, Firmin Didot, 1844, p. 27, parle à propos de Jugurtha, des «brusques évolutions que les anciens admiraient et que nous admirons encore chez les cavaliers africains ». Sur le goût d'Abdelkader pour les chevaux : B. ÉTIEnne, Abdelkader, Paris, Hachette, 1994, p. 67-72. Une passion qu'il partage avec le consul Daumas dont il corrigera le traité d'hippologie ; ibid., p. 141.

30 Cité dans Abdelkader, Écrits spirituels, Paris, Seuil, 1982, p. 15-16. Pour l'expression de " moderne Jugurtha ", voir la lettre de Bugeaud à Thiers du 5 avril 1843, Par L'épée et la charrue. Écrits et discours de Bugeaud, Paris, PUF, 1948, p. 139 : "Tant que nous n'aurons pas réduit à presque rien le moderne Jugurtha..."

31 En 1852, "La complainte d'Abdelkader " reprise par les journaux parle aussi du " moderne Jugurtha »: B. ÉTIENNE, Abdelkader, p. 248. La comparaison devient rapidement une banalité inévitable : "Ainsi finit celui qu'on a appelé justement l'Abdelkader de l'Antiquité » (É. CAT, Histoire de l'Algérie, Alger, Jourdan, 1880, p. 56) ; "Cette guerre nous montre le caractère des indigènes tel que nous le trouverons à toutes les époques, qu'il s'agisse de soutenir Jugurtha, Tacfarinas... ou Abdelkader » (E. Mercier, Histoire de l'Afrique septentrionale, Paris, Leroux, 1880, I, p. 67). 
confie sa nation à l'ordre français reconnu comme porteur d'avenir ; il se retourne évidemment contre la France si l'on perçoit la résistance de l'émir comme justifiée face à une armée qui n'a rien à faire en Algérie ${ }^{32}$. Cette ambiguïté, qui permettra sa récupération nationaliste sous des modalités que nous verrons, se retrouve parfaitement, par exemple, dans le fameux poème de Rimbaud, écrit en $1870^{33}$. Abdelkader n'y est pas directement nommé, mais il se profile derrière l'évocation de son descendant, "ce nouveau Jugurtha " qui, s'il " croupit, enchaîné, dans une indigne prison ", avait auparavant exhorté à défendre la nation et la patrie afin que "le Français ne déshonore plus les rivages arabes". L'enthousiasme national du héros reçoit sa force de celui de Rimbaud, et la strophe finale, lui recommandant de s'abandonner à la générosité de la France, n'en paraît que plus décalée, avec le recul du temps, au regard de l'exaltation patriotique précédente ${ }^{34}$.

Néanmoins, dans cette perspective ambiguë, ce Jugurtha-Abdelkader qui s'est enfin soumis, lui, à la différence du personnage sallustéen, peut aussi devenir, chez le colonisateur, un support à la construction de l'imaginaire collectif d'une «nation " qui accède à l'existence, si l'on peut entendre ainsi l'utopie parfois entretenue de plusieurs communautés enfin réunies en Algérie sous la haute autorité de la France, héritière de Rome. Le Jugurtha des nationalistes algériens est-il plus univoque ? Et quels liens entretient-il avec le précédent et avec le modèle antique ?

C'est, bien entendu, L'éternel Jugurtha de Jean Amrouche, rédigé en plusieurs étapes de 1943 à 1946, et publié cette même année dans la revue L'Arche, qui constitue l'étape décisive de la carrière contemporaine du personnage de Salluste, élargissant l'utilisation aux milieux colonisés ${ }^{35}$. L'essai se présente comme une réfutation du stéréotype du berbère, non pas en le niant, purement et simplement, mais en retournant positivement les défauts qu'on lui imputait.

Jusqu' ici, la charge symbolique double de Jugurtha comme représentant de l'éternel Numide et comme opposant irréductible à la conquête n’avait été définie et utilisée que

32 Et cela même si B. ÉTIENNE indique bien par ailleurs la difficulté à trouver un équivalent arabe au concept de «nation » dans le lexique utilisé par l'émir : Abdelkader, p. 120 et 168. Le terme bilad renverrait à la patria latine au sens de lieu des racines ; al-Watan utilisé au $\mathrm{xx}^{\mathrm{e}}$ siècle avec le sens actuel de " patrie " désignait à l'époque le territoire de la tribu.

33 CEuvres complètes, Correspondance, Paris, Bouquins, Laffont, 1992, p. 16-21.

34 Sans compter qu'au moment où écrit Rimbaud Abdelkader n'est plus prisonnier depuis longtemps. La grande popularité de l'émir en France même et l'admiration qu'il suscite tiennent en partie à son rôle de héros national : cf. une médaille de Bovy de 1862 à l'effigie de l'émir et portant au revers la mention : ÉMIR DE L'AFRIQUE DU NORD. DÉFENSEUR DE LA

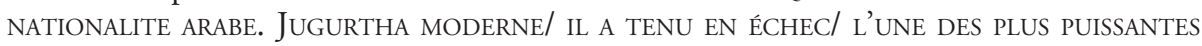
NATIONS/ DE LA TERRE.

35 J. Amrouche, L'éternel Jugurtha, consulté ici dans Algérie. Un rêve de fraternité, textes choisis et présentés par G. Dugas, Paris, Omnibus, 1997, p. 373 sqq. Sur la genèse de l'œuvre, J. Dejeux, Studi maghrebini, p. 129-131 ; biographie par R. LE BAUT, Jean El-Mouhoub Amrouche, Algérien universel, Paris, Alteredit, 2003, p. 47 pour la formation d'Amrouche. 
par le colonisateur pour illustrer d'une certaine manière la vocation du Berbère à subir le joug civilisateur et les obstacles que l'on allait rencontrer en chemin. La réflexion ambivalente sur Jugurtha n'était en fait que le monologue d'un conquérant qui s'interrogeait avec les outils intellectuels dont il disposait sur la nature et les chances de sa mission. Désormais, le mythe allait migrer d'un camp vers l'autre, avec un premier acte où la double identité de Jean Amrouche assume les personnages des deux adversaires à la fois. Berbère par sa naissance et sa langue maternelle mais de confession chrétienne, normalien, professeur de lettres et poète français autant que berbère, Amrouche algérianise le mythe avec toutes les ambiguïtés liées à sa propre pluralité identitaire. C'est pourquoi ce dialogue prend tout au long la forme d'une interrogation douloureuse sur la berbérité, une interrogation porteuse des ruptures de la double identité d'Amrouche irrémédiablement imprégné d'une culture classique qui l'oblige à assumer l'archétype du Numide sallustéen, et vivant par ailleurs comme un espoir celui de l'irréductible Jugurtha. Il est trop proche des auteurs latins pour évacuer d'un trait de plume l'archétype antique et limiter désormais Jugurtha, comme le fera l'idéologie nationaliste ultérieure, à une préfiguration des maquis algériens. Encore comme intimidé face à l'archétype dominant du Berbère, il hésite entre explication, autojustification et avertissement en demi-teinte, mais tout cela en maintenant toujours son discours au sein des catégories culturelles latino-françaises.

Quelles sont, en effet, ses références ? D’abord Salluste, bien sûr, et en ce sens, l'admirateur de la culture latine qu'était Amrouche n'avait pas encore coupé le mythe de sa source. Mais outre Salluste, le vocabulaire utilisé par Amrouche, certaines tournures de phrase montrent l'utilisation directe, ou presque directe si l'on considère qu'elles avaient fini par constituer une sorte de vulgate, des réflexions que Paul Monceaux avait exprimées sur le tempérament des auteurs latins d'Afrique en $1894^{36}$ et des analyses que Stéphane Gsell mettait en conclusion des deux volumes consacrées aux Berbères dans son Histoire ancienne de l'Afrique du Nord en $1925^{37}$. « Je suppose, pour plus de commodité, qu'il existe un génie africain ": ainsi débute Amrouche en écho à divers sous-titres de Monceaux, "Le génie africain et l'éducation classique » ou "Les éléments du génie africain ». Une analyse plus détaillée montre que ces "éléments " définis par Monceaux se retrouvent chez Amrouche : emphase, enflure, passion, goût de la joute, tentation de l'absolu, démesure, ivresse des images brillantes ${ }^{38}$. Les critères mêmes qui définissent de manière globalement positive l'auteur africain chez Monceaux font une partie

36 P. Monceaux, Les Africains. Étude sur la littérature latine d'Afrique. Les pä̈ens, Paris, Lecène et Oudin, 1894. J. Déjeux a noté l'importance des idées de P. Monceaux pour la construction du mythe de G. Audisio, mais curieusement ne l'utilise pas pour expliquer le Jugurtha d'Amrouche. Le thème de l'influence du sol et du climat africain sur le tempérament berbère, utilisé par J. Amrouche (et signalé par J. DéJEuX, op. cit., p. 129) est largement développé chez P. Monceaux.

37 Cf. supra, note 23. Cette fois, J. Déjeux note des " résonnances» (p. 133) entre S. Gsell et J. Amrouche. Il s'agit de bien plus que cela, d'une véritable réponse à $S$. Gsell.

38 P. Monceaux, Les Africains, chapitre I surtout, puis passim dans le reste de l'ouvrage. 
essentielle de l'âme berbère chez Amrouche. Il faut se tourner vers Gsell pour trouver le complément négatif de la description : caractère insaisissable, indiscipliné, apte au chaos, démesuré en tout et en particulier dans sa sensualité, cyclothymique, fuyant et faux, revêtant tour à tour comme des masques les traits de civilisation des divers conquérants. Les tournures de phrase utilisées par Amrouche renvoient aussi bien à Monceaux qu'à Gsell lorsqu'elles transforment l'exposé en un " manuel d'utilisation » du Berbère par le conquérant : «Ne confondez pas cette inactivité... avec la... paresse »; «si l'on ménage son amour-propre... on peut obtenir de lui... jusqu'au dévouement le plus passionné "; "Soyez éloquent, pressez-le de paroles émouvantes et obtenez de lui qu'il se range à votre avis ${ }^{39}$ \%. Dans ce manuel, Amrouche lui-même hésite à se situer comme le montre le jeu des pronoms nous/vous : "Nous voici affrontés au masque premier : le visage nu de Jugurtha... c'est à lui que vous avez affaire ${ }^{40}$. " C'est là une des dernières tentatives d'en faire un héros collectif pour une Algérie commune, mais la disjonction se profile. Si Amrouche a puisé ainsi à deux œuvres incontournables d'un historien et d'un spécialiste d'histoire littéraire qui furent les plus grands du moment, il n'a sans doute pas besoin de leur truchement dans ses recours aux Anciens pour ses réflexions sur la « mauvaise foi punique ${ }^{41}$ » et pas davantage pour approcher deux autres aspects importants de son Jugurtha. Son passage sur la superstition et la magie au Maghreb montre qu'il a bien en tête l'auteur ancien considéré comme l'Africain par excellence, Apulée, et son héros Lucius métamorphosé en âne, victime de sa curiosité pour la magie ${ }^{42}$. Mais surtout, l'image qu'il donne d'un Berbère en perpétuelle métamorphose civilisationnelle, insaisissable dans son goût du mouvement de la vie, fasciné avant tout par le brillant et l'éclat des mots et des choses, rejoint une définition du baroque par les thèmes de Protée et du Paon que l'on a pu proposer ${ }^{43}$. Or ce que G. Picard suggère d'appeler le "baroque africain " reste l'une des expressions privilégiées des spécificités culturelles de l'Afrique romaine et Apulée, revendiqué comme modèle par les écrivains africains ultérieurs, en constitue pour la région l'exemple littéraire par excellence. Sans le dire explicitement, mais en tournant autour de la notion, Amrouche perçoit le Berbère comme une éternelle métamorphose

39 J. Amrouche, L'éternel Jugurtha, p. 378-379. Pour le style "mode d'emploi » du Berbère à l'usage du conquérant, voir S. GSELL, op. cit. p. 284 : «Il faut que des étrangers viennent imposer à ce peuple l'ordre et la paix... Ils doivent être... ils se résigneront... Ils ne feront pas ; La nation dominante devra... elle invitera... elle trouvera... "; ou P. MonCEAUX, Les Africains, p. II-III.

40 J. Amrouche, L'éternel Jugurtha, p. 375.

41 Ibid., p. 380.

42 Apulée, Métamorphoses; et Apologie pour sa défense contre une accusation de magie.

43 Sur le baroque africain: G. C. PICARD, La civilisation de l'Afrique Romaine, Paris, Études augustiniennes, 1990, chapitre VI. On emprunte la métaphore de Protée et du Paon à J. Rousset, La littérature de l'âge baroque, Paris, Corti, 1953. 
qui s'adapte aux mouvances infinies de la vie ${ }^{44}$ et en ce sens on peut considérer que sa vision est fondamentalement celle d'un écrivain " latin » du Maghreb pour qui Apulée reste à travers le temps le grand ancêtre. Quant à la quête tourmentée de l'absolu, elle caractérise tout autant les autres grands Africains que sont Tertullien et saint Augustin que le Jugurtha d'Amrouche.

C'est ici l'Amrouche « latin » qui tente de réconcilier le Berbère avec le stéréotype du Numide, issu des textes anciens et utilisé par le conquérant français pour justifier sa présence. La mobilitas berbère n'est plus incohérence, versatilité et indiscipline, mais fidélité à soi-même en une constante adaptation aux mouvements de la vie.

Outre ce mythe de Jugurtha construit par Amrouche, J. Déjeux en étudiait deux autres : celui de l'éternel Méditerranéen chanté par Louis Bertrand et celui de l'éternel Méditerranéen "méditerranéen » (sic) de Gabriel Audisio et Albert Camus. Le premier, le mythe latin, reposait sur l'idée d'une Méditerranée fondamentalement latine, voire romaine $^{45}$; le second, plus universel, renâclant devant l'exclusivisme volontiers raciste et certains aspects régimentaires du mythe latino-latin lui préférait l'image d'un Ulysse cosmopolite et voyageur, d'un héros profondément sensuel, fils du soleil et de la Méditerranée, homme à deux visages, voire contradictoire, Janus, homo duplex ${ }^{46}$.

Ces deux mythes s'enracinaient dans la culture antique et on remarquera qu'en ce sens l'éternel Jugurtha d'Amrouche fait de même. Sur ce point les trois mythes sont parents et c'est d'un même fond culturel qu'émergent les divers héros. On peut même dire que c'est de Louis Bertrand que l'éternel Jugurtha est de ce point de vue le plus proche par ses sources essentiellement latines. Et c'est du sein même de cette perspective latine, et donc coloniale compte tenu de l'époque, qu'Amrouche présente la défense et les revendications de son Jugurtha.

Rien d'étonnant dès lors, si l'autre stéréotype constitutif de son mythe, celui de Jugurtha-héros national revendicatif s'exprime de manière aussi timide. Amrouche se limite à des recommandations modérées de reconnaissance du besoin de dignité, d'égalité et de liberté qui émanent du Berbère. Comme l'indique Déjeux : "On ne peut pas ne pas constater que l'auteur ne parle jamais de la conjoncture politique, du "colonialisme 47 ". " Il en cherche ensuite les raisons : " mystification " ? "Mytheopium »? Pour conclure que compte tenu de son assimilation, Amrouche ne peut proposer aux Berbères qu'un dépassement de soi vers la pensée et la technique occidentales et il y voit une limite du Jugurtha d'Amrouche qui ne peut être, dès lors, que partiellement mobilisateur dans la perspective du nationalisme algérien. Il suggère une

44 "Pour Jugurtha, vivre c'est épouser aussi étroitement que possible le mouvement, la durée, c'est rester souple, pour faire face aux circonstances changeantes, qui modifient sans cesse les conditions de l'action ", op. cit., p. 381.

45 J. Dejeux, Studi maghrebini, p. 70 sqq.

46 Ibid., p. 103 sqq. ; G. Audisio, Ulysse ou l'intelligence, Paris, Gallimard, 1945.

47 J. Dejeux, Studi maghrebini, p. 141. 
autre raison à cette faiblesse à partir du témoignage de personnalités qui ont connu Amrouche, Armand Guibert et Jean Daniel : « Son héros, tel qu'il le décrit, n'est guère "positif". Peut-être cela découle-t-il du fait que l'auteur est parti non d'une belle idée, mais d'un homme concret, lui-même, plus vrai que le portrait d'un ancêtre purement imaginé $^{48}$. " Le mythe deviendrait alors plus personnel que collectif. Les deux raisons alléguées par Déjeux, assimilation et projection personnelle, sont sans doute exactes, mais elles montrent surtout à quel point le recours à l'Antiquité par l'idéologie coloniale fut efficient. Amrouche a parfaitement intériorisé comme image de lui-même celle d'un Africain vaincu et largement négatif, issue de l'Antiquité, et dont le devenir ne peut se trouver que dans un dépassement de soi en direction de la francisation, comme l'Africain s'était accompli dans la romanisation. On comprend d'autant mieux sa violente réaction contre lui-même exprimée en 1959 dans ses carnets : "J'apprenais à vivre à la manière de mes maîtres, de qui j’avais fait miens les héros, les fables, les chansons et le langage... Un mimétisme de corps et d'esprit, qu'on reconnaît volontiers à ceux de ma race comme une disposition native particulière m'aidait dans ma tentative d'enracinement en une patrie distincte de ma patrie naturelle ${ }^{49}$. "Rien d'étonnant non plus à la méfiance que l'histoire nationaliste maghrébine manifeste parfois à l'égard de l'histoire ancienne ${ }^{50}$. L'image d'un Amrouche prisonnier de ses stéréotypes et déjà largement engagé dans le métissage culturel par le biais de la culture classique montrait qu'il existait bien là une séduction et un danger.

J. Déjeux note que "d'autres essayistes ou romanciers, engagés dans le combat nationaliste pour l'indépendance, ressusciteront eux aussi l'ancêtre mais pour une affirmation radicale de la rupture : Mohamed Chérif Sahli et Henri Kréa ${ }^{51}$ ». La radicalisation vient évidemment de l'avancée de la lutte pour l'indépendance. Et la rupture vient de la sélection, parmi les deux composantes du mythe, du seul élément nationaliste, largement étranger au Jugurtha latin de Salluste. On abandonnait l'image d'origine romaine autrefois principale, la perception antique du Numide, intériorisée par Amrouche et qui lui avait inspiré son Jugurtha. La colonisation avait suscité la nation algérienne, avait fourni par le biais de sa propre réception de l'Antiquité un héros national correspondant. Il restait en effet à en achever la réappropriation algérienne.

On reviendra donc rapidement, pour achever cet itinéraire de notre héros, sur Le message de Yougourtha de Mohamed Cherif Sahli, publié en 1947 comme « un livre de combat " destiné à " montrer que la liberté avait dans notre pays des racines... profondes ; (montrer) le dur et inévitable chemin de la lutte armée qu'avaient emprunté tant de générations et qu'allaient emprunter de nouveau avec succès les héros du

48 Ibid. et p. 136-137 pour l'autoportrait d'Amrouche en Jugurtha.

49 Ibid., p. 143.

50 Méfiance exprimée de manière particulièrement exemplaire par A. LAROUI, L'histoire du Maghreb. Un essai de synthèse, Paris, Maspéro, p. 21 sqq.

51 J. Dejeux, Studi maghrebini, p. 121-122. 
$1^{\text {er }}$ novembre 1954 ; (montrer) la nécessité d'une large union des forces patriotiques... d'une action rationnelle ${ }^{52}$ ». Le cadre de la récupération du héros est donc clairement indiqué dès le départ. Il rejoint celui qui est fixé par les chartes nationales successives du peuple algérien après l'indépendance : « Charte d'Alger » de 1964, puis chartes de 1976 et $1986^{53}$, à travers lesquelles on voit que Jugurtha constitue le symbole de l'éternelle résistance algérienne à l'impérialisme, renforçant la dynamique téléologique de l'histoire nationale. L'ouvrage de Sahli commence par revisiter l'histoire ancienne de l'Afrique du Nord avant Jugurtha. On y retrouve la perspective dominante de l'historiographie algérienne qui perçoit la nation comme une proie éternellement convoitée par des impérialismes successifs qui profitent des divisions de la société berbère. L'histoire algérienne est en fait une longue et tragique résistance au fil du temps, dont Jugurtha est donc l'initiateur et qui culmine avec la révolte de novembre 1954. Dans cette évocation d'une quarantaine de pages, la moitié de l'ouvrage, la phraséologie contemporaine est systématiquement appliquée à l'Antiquité : Carthage devient « un cancer impérialiste au flanc du Maghreb " et les révoltés de « la Guerre des Mercenaires » sont qualifiés de "patriotes tunisiens".

Mais que reste-t-il de Salluste dans la suite de l'essai de Sahli, lorsqu'il évoque l'épopée de son héros ? Juste une trame événementielle et deux citations textuelles un peu longues de l'auteur latin pour évoquer la description physique et morale de Jugurtha puis un assaut victorieux des Numides devant Zama ${ }^{54}$. Il serait par ailleurs bien difficile de retrouver après Sahli dans le texte sallustéen la phrase qui indiquerait que " les Numides ne peuvent être enchaînés ni par la crainte ni par les bienfaits »! Le reste est une reprise de l'événementiel à travers le prisme de l'analogie avec l'histoire contemporaine. Ainsi Jugurtha se voit-il attribuer par Rome " la province d'Oranie et l'Algérois 55 ". Plus tard, le Numide demande l'assistance de Bocchus " roi du Maroc ", mais « l'armée maghrébine (Algériens et Marocains réunis) » est vaincue. Suit la « trahi-

52 M. C SAHLI, Le message de Yougourtha, Alger, En-nahda, 1968 (2 édition), p. 5-7.

53 La Charte d'Alger, édition de la commission centrale d'orientation du FLN, Alger, 1964 ; texte de 1976 et commentaire dans R. Lambotte, Algérie. Naissance d'une société nouvelle, Paris, Éditions sociales, 1976. Jugurtha est "l'initiateur de la résistance à l'impérialisme romain " (1976, p. 83) ; à " la résistance populaire acharnée (contre l'impérialisme) s'attachent des noms tels que celui du véritable héros que fut Jugurtha » $(1964$, p. 10) ; «Sous la conduite de Jugurtha, le peuple a entrepris de résister à cette tentative de subjugation, et de défendre le patrimoine des ancêtres. Jugurtha s'est attaché à préserver l'unité de l'État et son indépendance et à arracher la Numidie à la tutelle de Rome. La poursuite des années durant de cette résistance face à la plus grande puissance de l'époque témoigne de la cohésion du peuple autour de Jugurtha, de son adhésion aux objectifs proclamés visant à la préservation des fondements de l'État et de l'indépendance de la décision. » (1986)

54 M. C SAHLI, Le message de Yougourtha, p. 42 et 57.

55 Ibid., p. 48. 
son " du souverain marocain, " un coup de poignard dans le dos ${ }^{56}$ ", qui évoque clairement l'abandon d'Abd El-Kader par le sultan du Maroc après leur défaite commune de l'Isly, puisque Sahli avait auparavant associé les deux personnages en remarquant : "Que le noble Abd El-Kader, vingt siècles plus tard, imitât son prédécesseur (en demandant l'aide "marocaine"), ce n'était pas une coïncidence ${ }^{57}$. "

Le combat nationaliste s'est ici largement coupé des racines latines, développant son propre Jugurtha. Salluste ne sert plus alors que de trame événementielle pour la construction d'un héros national : «Tombé en pleine lutte (Jugurtha) reste pour nous l'émouvant messager de cette grande espérance du cœur humain, qui se nomme liberté. De génération en génération, de siècle en siècle, son message a été le credo du peuple, le mot d'ordre des patriotes ${ }^{58}$. "Le rêve d'unité maghrébine sera en fait réalisé par les Almohades qui verront " la fusion du génie arabe et du génie berbère ${ }^{59}$ ». Cette phrase, comme celle où l'auteur indique que "l'islam est un admirable complément du génie africain ${ }^{60}$ » résonne, par la reprise du terme de "génie », comme un écho affaibli de l'historiographie coloniale au cœur de l'affirmation d'une identité algérienne arabo-musulmane.

Nous terminerons cette étude de la réception contemporaine de Jugurtha sur une allusion à son portrait monétaire. De manière étonnante, ce roi ne nous a laissé aucune effigie, ni même aucune émission à son nom. Cet étrange silence qui contraste avec la force du témoignage de Salluste a suscité l'imagination des numismates et certains lui ont assigné des portraits en déshérence sur des émissions difficiles à attribuer. C'est le cas de Lüdwig Müller, qui en 1870, lui avait donné une effigie maintenant correctement identifiée comme étant celle de Melqart (fig. 1) ${ }^{61}$.

56 Ibid., p. 61. L'analogie était faite par l'historiographie coloniale dès 1880 par E. MERCIER, Histoire de l'Afrique septentrionale, Paris, Leroux, tome 1, p. 40 : "Telle fut la chute du Jugurtha moderne. De même que pour l'ancien, c'est grâce à la coopération du roi de Maurétanie, son ancien allié, qu'elle fut obtenue. "

57 Ibid., p. 62.

58 Ibid., p. 68.

59 Ibid., p. 12 et 76.

$60 \quad$ Ibid, p. 8.

61 L. Müller, Numismatique de l'Ancienne Afrique, Copenhague, 1870, III, p. 34-35. Pour l'identification correcte : G. K. Jenkins, Sylloge Nummorum Graecorum. The Royal Collection of Coins and Medals, Danish National Museum, North Africa, SyrticaMauretania, Copenhague, $1969, \mathrm{n}^{\circ} 382-383$. Un point de la question par L. I. MANFREDI, Monete puniche, repertorio epigrafico e numismatico, Bolletino di numismatica, monografia 6, 1995, p. 210, répertoire SIB, 5-6. 


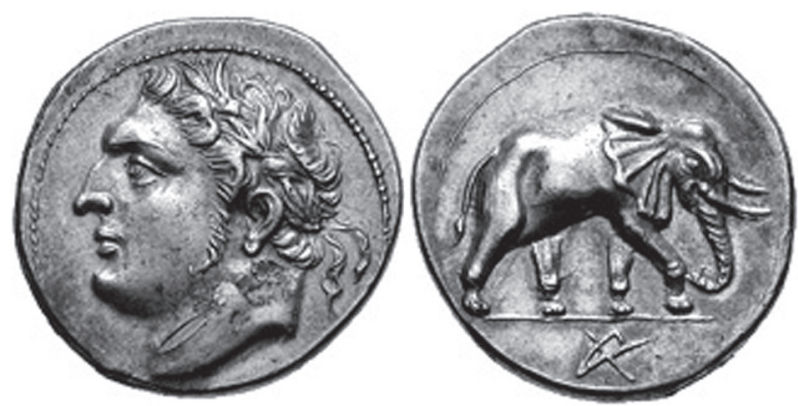

Figure 1 (Photo CNGcoins)

On utilise plus rarement, dans le même rôle, une pièce d'argent frappée en Espagne durant la deuxième guerre punique, qui montre à l'avers une effigie de Melqart ou d'Hannibal, au revers un cheval et un palmier symboles de Carthage (fig. 2) ${ }^{62}$.
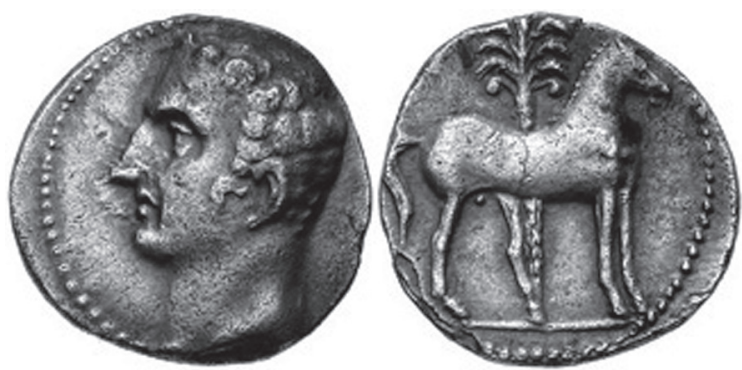

Figure 2 (Photo CNGcoins)

Le premier " portrait de Jugurtha » est encore largement répandu en Algérie, aussi bien dans les travaux d'historiens que dans ceux de vulgarisation ou les manuels scolaires ${ }^{63}$. Le second est repris sur une émission tunisienne de prestige, surmontant une carte de la Numidie et une balance dont les plateaux portent à gauche la louve romaine et à droite une monnaie de Carthage au palmier (fig. 3).

62 G. K. Jenkins, Sylloge Nummorum Graecorum, no 295.

63 Deux exemples récents : M. KADDACHE, L'Algérie des Algériens, de la Préhistoire à 1954, Paris, Edif, 2000, p. 54 ; M. A. Haddadou, Les Berbères célèbres, Alger, Berti, 2003, couverture de l'ouvrage. 


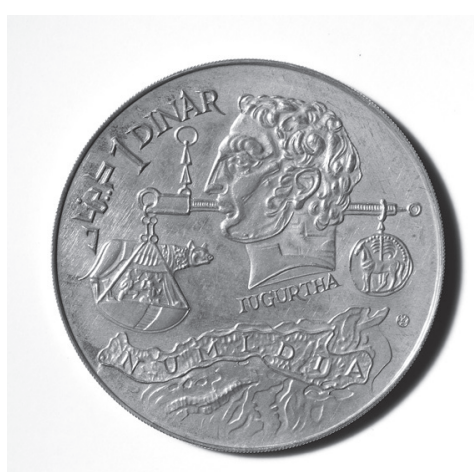

Figure 3

Jugurtha tient donc la balance du destin entre Rome et l'Afrique, mais une Afrique aux couleurs de Carthage puisque le motif de la monnaie est punique et non numide. La Tunisie se réclame du héros berbère, mais dans un cadre civilisationnel carthaginois qui garantit la spécificité " tunisienne » du héros. Par ailleurs, le plateau "romain " de la balance se trouve à l'ouest, le plateau " punique " à l'est, inversant l'ordre des réalités géographiques au profit d'un ordre symbolique : Rome représente l'Occident et Carthage l'Orient. Au-dessus, Jugurtha semble alors curieusement vouloir tenir un équilibre entre les deux plateaux, à l'image de la politique de Bourguiba qui se voulait " un Jugurtha qui a réussi ${ }^{64}$ ". Ce qui, après tout, reste fidèle à l'un des aspects paradoxaux de Jugurtha héros national : un certain rôle de médiation, par-delà les récupérations, entre colonisateurs et colonisés.

On voit que la rupture effectuée par l'idéologie nationaliste avec les sources littéraires latines et le choix d'un Jugurtha essentiellement national ne supposent évidemment pas que l'on renonce à parer le héros du prestige d'un ancrage antique ${ }^{65}$.

L'attention que nous avons portée à quelques jalons de l'itinéraire de Jugurtha à travers le temps nous ramène en conclusion aux conditions d'une histoire partagée du Maghreb antique. Si on laisse de côté des positions limites selon lesquelles l'Antiquité n'est d'aucun ou que de peu d'intérêt pour la constitution ou la compréhension des identités maghrébines contemporaines, on voit que la récupération analogique a fonctionné aussi bien dans tous les camps idéologiques pour expliquer, légitimer et dénoncer. Le personnage de Jugurtha s'y prêtait particulièrement bien dans la mesure où il

64 Cf. notre article "Bourguiba et l'Antiquité : le témoignage des monnaies ", Mélanges Robert Sablayrolles (sous presse).

65 Une émission algérienne de 1994 à l'effigie de Jugurtha (C. KRAuse-C. Mishler, Standard Catalogue of World's Coins, $35^{\mathrm{e}}$ édition, 2008, $\mathrm{n}^{\circ} 134$ ) reste beaucoup plus sobre : une simple effigie stylisée à partir de l'habituel "portrait de Jugurtha » des monnaies antiques. 
avait été créé d'emblée avec assez de précision et de vague à la fois pour servir successivement à la formation de plusieurs imaginaires collectifs successifs liés aux conquêtes impériales comme à la résistance qu'on leur oppose. En l'occurrence, une différence parmi d'autres tient aux sources mises en valeur. L'époque coloniale privilégiait le texte antique où elle puisait dans la fidélité à l'écrit un schéma explicatif des lieux et des hommes. La lutte anticoloniale puis l'époque des indépendances se défient de l'autorité du texte vis-à-vis duquel elles prennent leurs distances, n'empruntant que tel ou tel morceau choisi voire un élément largement recréé. On lui préfere une trace interne à la berbérité, un portrait monétaire largement diffusé malgré toutes les incertitudes qu’il comporte sur son authenticité. Outre une suspicion vis-à-vis des écrits de l'adversaire vainqueur, qui ont nourri au fil du temps la culture de son « héritier " colonial, outre une tradition culturelle évidemment plus étrangère au latin, il faut y voir la nécessité d'un symbole tangible, mobilisateur, le besoin de donner un visage à ce Jugurtha afin de pouvoir l'inscrire en tête du martyrologe de l'indépendance algérienne. La disjonction du personnage de Jugurtha, entre celui des textes et le héros essentiellement ramené à un symbole contemporain, accompagne et nourrit l'évolution de Jean Amrouche dont l'identité biculturelle a procédé à l'ultime tentative de conciliation des deux approches. Cette attitude relève peut-être de ce que l'auteur a ensuite regretté comme un inutile asservissement de sa part. Quoi qu'il en soit, ces réceptions différentes et instrumentalisées, si elles n'empêchent pas de belles et fructueuses coopérations archéologiques bilatérales, expliquent peut-être en partie que la dernière histoire "générale " du Maghreb incluant l'Antiquité et rédigée en français par un historien, celle de Ch. A. Julien, soit quasi nonagénaire et arrête son étude à l'année 1830 !

Jacques AleXandropoulos

Université de Toulouse II-Le Mirail

PLH-ERASME (EA 4601)

Pavillon de la Recherche

5, allées Antonio Machado

F-31058 Toulouse Cedex 9

alexandropoulos.jacques@univ-tlse2.fr 